\section{Arqueología y rehabilitación en Sevilla. Desarrollo metodológico y práctico}

Miguel Ángel Tabales Rodríguez

Universidad de Sevilla

\section{INTRODUCCIÓN}

En Andalucía, como en el resto de España, se advierte un cambio radical en la concepción de la A rqueología desde mediados de los años ochenta, al recibir la comunidad autónoma las transferencias en materia de gestión e investigación. Junto a este hecho, cuya consecuencia principal fue la multiplicación de actividades sistemáticas y de urgencias, hay que destacar un considerable aumento de las operaciones de rehabilitación de edificios históricos que serían destinados a albergar organismos públicos.

Esta dinámica se vivió especial mente en la ciudad de Sevilla donde se aunaron distintas circunstancias que desembocaron en un aumento considerable de investigaciones arqueológicas "de apoyo a la rehabilitación"; las causas principales fueron el crecimiento urbano natural propio de la capital, el acondicionamiento de numerosos inmuebles como sede de la Exposición U niversal de 1992, la política de recuperación de grandes edificios históricos como sedes de delegaciones, consejerías y distintos servicios de la Junta de Andalucía y de la Gerencia de U rbanismo, etc.

Como resultado de todo ello el análisis arqueológico de conjuntos monumentales de considerable tamaño y complejidad (Conventos, palacios, iglesias, cuarteles, hospitales, etc.) se tradujo en el desarrollo de sistemas analíticos, basados en experiencias italianas (PARENTI, 1988: 249-279; BROGIOLO, 1988) y nacionales (CABAllero, 1985: 27-32; J IMÉnez, 1992; LóPez, 1984: 19-21) capaces de garantizar un nivel razonable de conocimiento edilicio, histórico y urbano, a la par que una razonable inserción arqueológica en los procesos de rehabilitación, desde las fases preproyectuales.

Las consecuencias de dos décadas de trabajos se traducen hoy día sobre todo en una aceptación "política y administrativa" de dichos procesos, habiéndose pasado de las complicadas excavaciones forzadas en inmuebles en pleno proceso de obra (al go común en los 80 ) a la integración de la cautela "A nálisis arqueológico de paramentos" en los planes de protección urbanísticos para cual quier edificio histórico.

\section{ARQUEOLOGÍA DEL EDIFICIO EN SEVILLA}

Para entender el auge de la nueva disciplina en nuestra ciudad debemos retroceder a las décadas centrales del siglo $X X$, momento en el que se materializan algunas rehabilitaciones de cierto calado en su casco histórico y en edificios emblemáticos de su pasado islámico. Estas pueden enmarcarse dentro de una visión historicista y violletiana de la restauración típicas de ese momento de Paradores

Key words: Seville, Archaeology of Buildings, Rehabilitation. 
$N$ acionales y reconstrucciones monumentales. Sus autores forman parte de una exquisita y reducida "casta" de arquitectos-historiadores formados dentro de la tradición erudítica de carácter histórico artística y especializados en la rehabilitación de monumentos islámicos; representaban un perfil (hoy muy escaso) de arquitectos como Félix Hernández o Rafael Manzano capaces de proyectar y restaurar con una comprensión de la edilicia histórica y de los lenguajes formales del pasado muy superior a lo habitual en la actualidad. A ellos debemos el descubrimiento, mantenimiento y recuperación de construcciones islámicas como el Patio de Crucero de la casa de Contratación de Sevilla, Patio del Yeso o el Sahan de la Mezquita Mayor Almohade, que nos hablan de una época (hasta 1985) en la que la $\mathrm{H}$ istoria del Arte y la Arquitectura se aunaban en los procesos de rehabilitación en un momento en el que aún no existía lo que hoy denominamos A rqueología de A poyo a la Rehabilitación y mucho menos Arqueología del Edificio. Son de este período algunas importantes excavaciones en monumentos locales como el Alcázar (Bendala, Negueruela, 1981: 335-379) o la Catedral (Collantes De Teherán, 1977) en las que ya se advierten las lógicas y habituales complicaciones metodológicas resultantes de las primeras aproximaciones de arqueólogos clásicos a los medios urbanos pluriestratificados.

Por entonces, años setenta, se renovó en la Escuela Técnica Superior de Arquitectura la asignatura "A nálisis deFormas A rquitectónicas" gracias a los arquitectos Pedro Rodríguez y Alfonso J iménez, quienes diseñaron un sistema de aproximación al edificio caracterizado por estudios ti pológicos extremadamente rigurosos cuya final idad era el conocimiento exhaustivo y sistemático del inmueble desde ópticas distintas: masiva, funcional, tecnológica y espacial. Un ejemplo magnífico del sistema fue puesto en práctica en 1977 en la Puerta de Sevilla de Carmona, publicada por Alfonso Jiménez en 1989. Dicho sistema se basaba en la tradición erudítica tradicional de la arqueología romana representada por Lugli y J ean Pierre A dam, desarrollada en el campo arqueológico desde aquellos momentos por Bendala y posteriormente Lourdes Roldán. Las posibles carencias del análisis tipológico se vieron complementadas por una excavación arqueol ógica de apoyo a la restauración que determinó la existencia de un amurallamiento del Bronce Final-Orientalizante (siglo VIII a.C.) bajo el actual sistema defensivo, que completaba una imagen ya bastante compleja dada la superposición de muros y espacios desde el período cartaginés al almohade. El conjunto tuvo su máximo esplendor durante el siglo I a.C., momento en el que se aprecia la erección de un sistema de acceso con intervallum y un templo sobre la terraza del bastión principal.

Esta experiencia, a pesar de haber sido emblemática en su momento y haber servido de referencia durante años gracias a su depurada metodología, no tuvo un desarrollo posterior completo en otros edificios o restauraciones y esto a pesar de que la asignatura de Análisis de Formas posibilitó a cientos de arquitectos que hoy trabajan en nuestro entorno las herramientas adecuadas para su material ización en programas de restauración. Fueron de nuevo los citados investigadores los que en las décadas siguientes acometieron aproximaciones similares en inmuebles como el H ospital de las Cinco Llagas, hoy Parlamento Andaluz, Catedral o I glesia de la M agdal ena.

En este foro de Vitoria, que ha supuesto uno de los intentos más serios de definición de una disciplina como la A rqueología de la A rquitectura en nuestro país, nos parece imprescindible reivindicar el carácter pionero de esta experiencia que fue coetánea de las primeras aplicaciones del método $\mathrm{H}$ arris sobre la edificación en Europa. De hecho los dibujos paramental es y reflexiones arqueológicas de Jiménez sobre la transformación muraria ya dejaban entrever un salto que no llegó a producirse, en nuestra opinión, por la escasa adhesión al uso de dicho sistema entre los arqueólogos locales clásicos durante los setenta. En cual quier caso, Ios resultados obtenidos en Carmona pusieron en evidencia la necesidad de complementar la rehabilitación con informes arqueológicos sobre el subsuelo y lo emergente, dejando atrás la etapa de los informes artísticos introductorios, algo presente en los debates que se desarrollaban en Italia en dicha época.

A partir de 1985 se produjo un incremento extraordinario de la rehabilitación de edificios históricos en la ciudad debido a tres circunstancias principales:

- Exposición Universal de 1992 y consecuente recuperación de inmuebles destinados a albergar eventos: M onasterios de la Cartuja y San Clemente, etc.

- Transferencias a la Comunidad Autónoma, que decide restaurar viejos edificios para instal ar consejerías y del egaciones, en especial la de Cultura en diferentes palacios del barrio de San Bartolomé (antigua judería).

- Impulso urbanístico desde la Gerencia Municipal de Urbanismo: Murallas islámicas, Monasterio de San J erónimo, etc.

Las primeras rehabilitaciones de envergadura coinciden con la creación de un servicio de arqueología en la Delegación Provincial de Cultura dirigido sucesivamente por Fernando A mores y J uan M anuel Campos, y posteriormente por J osé Manuel Rodríguez, quienes impul saron y 
forzaron la presencia arqueológica en las obras de rehabilitación en un contexto de excavaciones urbanas de urgencia cada vez más numerosas y complejas. Desde los distintos servicios de la Dirección General de Bienes Culturales se impulsó esta presencia en un ambiente de notable actividad e innovación (Creación del Instituto Andaluz del Patrimonio Histórico, Ley del Patrimonio Histórico Andal uz, Reglamento de Actividades A rqueológicas, etc.)

En las principales intervenciones (Cartuja, dirigida por F. A mores, o los Palacios de A Itamira, M añara, etc. por D. Oliva) se fue poniendo de manifiesto la necesidad de generar sistemas de trabajo específicos para la comprensión de la arquitectura antigua y su recuperación, pasándose de la excavación desvinculada del edificio a la creación de model os ágiles de trabajo integrados de manera razonable en los proyectos de rehabilitación en los que se atendía prioritariamente a la edificación en sí a la par que se diversificaban los campos de anál isis. Todo ello sucede, como en otras zonas de España de manera autónoma e intuitiva, a medida que comienzan a trascender las novedades científicas generadas en Italia (Brogiolo, Parenti, Doglioni, Francovich...) y las españolas (Caballero, López Mullor, etc.).

En los años noventa la actividad metodológica del Instituto Andaluz del Patrimonio y de otros grupos locales se tradujo en la aparición de escritos referentes a dichos sistemas de investigación (OJEDA, TABALES, 1996: 41-52), (TABALES, 1997: 65-81), e incluso alguna tesis de Licenciatura sobre M etodología arqueológica de apoyo a la rehabilitación (TABALES, 1993) o la tesis doctoral denominada Arqueología y rehabilitación de edificios históricos en Sevilla (TABA LES, 1998).

\section{LA REHABILITACIÓN EN SEVILLA}

Desde el punto de vista cronológico "creemos" que todas estas real idades han incidido en el panorama de la recuperación del patrimonio arquitectónico sevillano según el siguiente esquema:

- P roceso 1. 1985-1990. Se caracteriza por la realización de investigaciones parciales de edificios, general mente condicionadas por su inserción a posteriori en los procesos de obra de rehabilitación, y realizadas por lo general con una visión aún muy parcial de los estudios sobre el patrimonio edificado. Comúnmente, consisten en meras excavaciones realizadas "en el edificio", pero no necesariamente con éste en el primer orden de prioridades (aunque después se intente completar la fal ta de atención a la estructura emergente con anál isis por lo general superficial es y de carácter documental 0 artístico, del conjunto del monumento en cuestión). Metodológicamente son realizadas cada una según la formación del investigador y sus afinidades y general mente no se rigen por el sistema de distinción de unidades estratigráficas. A este primer grupo, que coincide con nuestros primeras excavaciones en la ciudad de Sevilla pertenecen las rehabilitaciones del N oviciado de San Luis, El Convento de San Agustín, Miraflores, M onasterio de San J erónimo, etc.

En dichas intervenciones se produjo en los arqueólogos implicados una transformación de los conceptos sobre A rqueol ogía incul cados por la U niversidad manifestándose una contradicción entre el interés científico personal, el interés científico del yacimiento urbano per sé, las necesidades de la rehabilitación, los condicionantes temporales y presupuestarios, etc. Difícil panorama al que se añadía el interés político y la falta de atención a otros edificios restaurad os por entonces. Los resultados de aquell las restauraciones, siempre parciales, vistos desde la distancia, fueron aceptables y en todas las intervenciones se detecta una aproxi mación intuitiva a los al zados aunque no se les aplica ni la misma atención que al subsuelo ni se le otorga el mismo rango científico. Son trabajos en edificios de grandes dimensiones en los que abundan los cortes puntuales y zanjas prospectivas; las áreas de excavación en extensión son poco frecuentes y se recurre a la vigilancia de movimientos de tierra con maquinaria. La lógica dicta las fases arqueológicas a val orar, que frecuentemente derivan en el redescubrimiento de ámbitos medievales o modernos derruidos.

- Proceso 2. 1990-1992. Caracterizado por su intención de análisis integral, con aspiraciones de asumir desde la óptica arqueológica el resto de los estudios multidisciplinares, general mente bien integrados 0 aceptablemente insertados en el proyecto de rehabilitación. Son los estudios de la Cartuja y del Palacio de A Itamira. A este grupo se pueden añadir en los años siguientes los del Palacio de M añara, el Cuartel del Carmen, Atarazanas, Monasterio de San Clemente, etc.

El salto cualitativo es notable durante este período y las experiencias en la Cartuja a cargo de Fernando A mores supondrán, además de una cantera de futuros arqueól ogos urbanos, un escaparate mundial y una reivindicación de la Arqueol ogía de la A rquitectura como disciplina necesaria en la toma de decisiones sobre rehabilitación en un grado no superado hasta el momento en nuestra comunidad. Debemos destacar la figura de Diego Oliva, conservador del Museo Arqueológico de Sevilla, quien defiende y pone en marcha la creación de grupos multidisciplinares y la figura del arqueólogo-historiador del edificio como alter 
ego del arquitecto diseñador (Oliva, 1993). Dicha figura, muy criticada desde el campo de la A rquitectura tuvo en dicho investigador a su mejor exponente durante el período en el que la reivindicación de la lectura arqueológica de los inmuebles era tema de discusión habitual en los foros universitarios y políticos andaluces. Sus trabajos en los Palacios de Atamira (1988), Mañara (1990), Marchelina (1995) y el Cuartel del Carmen (1993) son ante todo una muestra de integración y diversificación del campo de análisis arqueológico tradicional a la vez que un intento de integración en el programa de recuperación del inmueble, lej os de la lectura independiente y distanciada de la obra. - P roceso 3. 1993-1995. U tilización de un sistema de trabajo específico, basado en las experiencias anteriores y destinado a suplir las carencias detectadas previamente, mediante la especialización del equipo de investigación básico y la definición de aquellos aspectos esenciales para la caracterización global del inmueble.

Metodológicamente se caracterizó por su carácter experimental, poniéndose en práctica nuevos sistemas de registro, indagándose en diversos tipos de estudios paramentales. Pertenecen al grupo los trabajos en el Convento de Santa María de los Reyes, el Cuartel del Carmen, el Palacio de los $M$ arqueses de $M$ archelina, etc.

- Proceso 4. 1993-2002. A ceptación generalizada de la presencia arqueológica en edificios en rehabilitación. Creación de programas de rehabilitación por parte del Servicio de Conservación de la DGBC en iglesias mudéjares (A. Pérez Paz), creación de fichas diagnóstico para la conservación (Arturo Pérez y otros...). Participación de diversos organismos públicos en la rehabilitación de conventos y palacios (Consejería de Obras públicas en el Convento de Santa M aría de los Reyes, G erencia de U rbanismo en Santa Inés, etc.). Incorporación de la empresa privada (Palacio de San Leandro).

- Proceso 5. 1996-2002. Organización de equipos arqueológicos sistemáticos para la investigación y rehabilitación de grandes conjuntos monumentales en la Catedral, el Alcázar o el Antiguo Hospital de las Cinco Llagas.

- Proceso 6. En la actualidad la mayor aportación consiste en la incorporación de la arqueología del edificio en los Planes Especial es de Protección U rbana. M ediante la utilización de una cautela referente a la lectura de paramentos en dos modal idades (general o básica) se añade la estructura edificada al subsuelo, cautelado a nivel general desde 1995. Esta decisión se entiende dentro de un proceso anual de excavaciones de urgencia cercana a la cincuentena y en un momento de renacimiento de la actividad inmobiliaria.
La Gerencia de U rbanismo del Ayuntamiento de Sevilla ha sido una de las pioneras en Andalucía en la protección del Patrimonio A rqueológico Emergente, mediante la aplicación de una cautela específica contenida en el planeamiento urbanístico de protección de su Conjunto $\mathrm{H}$ istórico. Dicha cautela, denominada Análisis Arqueológico de Estructuras Emergentes, puede abarcar varios grupos de edificios, que, individualizados en las respectivas fichas de los Catálogos, al canzan tanto a los inmuebles considerados o declarados monumentos, como a todo aquella arquitectura de menor entidad que pueda contener cualquier elemento relevante de carácter patrimonial (estructuras más antiguas que las aparentes, su propia cronología, etc.). EI grado de protección establecido dependerá del nivel y tipo de obra, que, igualmente, basculará entre el estudio integral del edificio, en casos de obras generales, como el análisis mínimo de la tipología edilicia, mediante la documentación de el ementos constructivos y los espacios que la conforman. Para todos estos casos, la Gerencia de U rbanismo ha establecido un procedimiento administrativo vinculado a la licencia de obras, y su seguimiento correspondiente mediante una inspección técnica municipal. H asta diciembre de 2001, cerca del $10 \%$ de las intervenciones arqueológicas que se real izaron bajo tutela local contenían esta cautela1.

- Proceso 7. Presencia de la U niversidad como institución en los trabajos de apoyo a la rehabilitación o en la docencia de los métodos oportunos. Ya iniciada durante los ochenta por Fernando Amores en el Departamento de Prehistoria y A rqueol ogía y complementada por cursos de doctorado y Masters. En el curso 2001-2002 se ha puesto en marcha una nueva asi gnatura: "A nálisis arqueológico de edificios en rehabilitación", de libre configuración, diseñada para arqueólogos, arquitectos técnicos y arquitectos, impartida en la Escuela de A rquitectos Técnicos.

\section{UNA ESTRATEGIA DE INTERVENCIÓN}

En diferentes trabajos anteriores se ha incidido sobre aspectos vinculados al proceso de intervención arqueológica en inmuebles, desarrollando un sistema metodológico diseñado para ese fin por nuestro equipo (TABALES, 1993). No significa esto que todos los investigadores sevillanos hayan seguido ni aceptado dicha propuesta pero el número de rehabilitaciones en las que ha sido puesta en prácti-

${ }^{1}$ En esta novedosa y esperanzadora experiencia ha tenido un papel determinante el arqueólogo de la G erencia de U rbanismo O scar Ramírez a quien agradecemos su ayuda para la realización de esta síntesis. 
ca y su uso casi exclusivamente local nos permiten divulgarla como una consecuencia del proceso antes expuesto2.

En síntesis, el citado sistema se basa, desde el punto de vista de la organización y dejando al lado los fundamentos metodológicos, en las siguientes pautas:

1- Acercamiento inicial al edificio.

- División zonal, identificación de "unidades-guía”y estancias.

- Estudio previo y pormenorizado de la estructura emergente.

- Estudio de los sistemas de adosamiento y contacto entre alineaciones.

- A nálisis edilicio provisional (constatación de los tipos de muro).

- Realización de un programa de Muestreos edilicios.

- Cumplimentación de las observaciones tipológicas.

- Registros de el ementos artísticos o susceptibles de conservación.

- Elaboración de una hipótesis evolutiva inicial.

2- Análisis de paramentos.

- Estudios estratigráficos.

- Análisis tipológicos.

- Constatación de los principal es eventos estructurales.

3- Estudio del subsuelo.

- Realización de cortes estratigráficos.

- A pertura de cortes y zanjas-guía.

- Realización de cortes con carácter "extensivo"

\footnotetext{
${ }^{2}$ Algunas investigaciones real izadas en inmuebles sevillanos por nuestro equipo: Cuartel del Carmen 1990-94, Monasterio de San Clemente 1991-1992, Convento de Santa María de los Reyes 1992, Palacio de San Leandro 1992, Pal acio de M archelina 1995, Catedral 1996-1998, A lcázar 1997-2002, H ospital de las Cinco Llagas 1998-2001. Otras intervenciones fuera de la ciudad: I glesia de Santa Ana de Guadal canal 1995-1996, Puerta de Córdoba en Carmona 1995, etc. Investigaciones realizadas por el autor de estas páginas junto a $\mathrm{F}$. Pozo (C. Carmen), D. Oliva (C. Carmen), Reyes Ojeda (P. Córdoba), Arturo Pérez (S. Leandro), Rosario Huarte, A. Jiménez y Pablo Oliva (Catedral, Hospital C. Llagas y Alcázar), Carmen Romero (Santa A na), etc.

Otras intervenciones previas fueron determinantes para la generación del siste ma: Palacios de Altamira 1988 (E. Larrey y D. Oliva), Monasterio de San J erónimo (Pozo 1987), Castillo de Alcalá de Guadaira (M.A. Tabales y F. Pozo), Cartuja (Am OREs et alii, 1988-1992), Palacio de Mañara 1989-1991 (D. Oliva, S. Rodríguez, R. Ojeda y M.A. Tabales).

La lista de investigadores en programas de rehabilitación durante estos años en la ciudad es aun mayor destacando el magnífico trabajo de Cruz A gustina Quirós en las Reales Atarazanas desde 1990, o Antonio Pérez Paz en iglesias de la Magdalena, San Bartolomé, San Esteban, San Julián, y en especial en la I glesia de San Andrés, o las investigaciones de Manuel Vera en la Buhayra y en el castillo de San J orge, donde participaron igual mente $\mathrm{M}$ arc $\mathrm{H}$ unt y otros renombrados arqueólogos: C. Romero en Miraflores. Otros investigadores como I sabe Santana, Sandra Rodríguez, Oscar Ramírez, José Escudero, Teresa M oreno, F. M endoza, etc. han contribuido notablemente a la implantación de esta disciplina en los programas urbanos. Aún a riesgo de olvidar a al gún compañero, no podemos terminar esta breve recesión sin elogiar la labor pionera de J. Manuel Campos, Fernando Amores y José Manuel Rodríguez, quienes desde la Delegación Provincial de Cultura impulsaron la arqueología del monumento. La lista de responsables en los distintos servicios de la D G BC es importante por lo que debe excusarse una mención tan genérica como la presente
}

4- Control de Obras.

- Control de las actividades de restauración.

- Control de las obras de rehabilitación.

En relación con el REGISTRO Y MÉTODO:

a- Seguimiento del método $\mathrm{H}$ arris con ciertas puntualizaciones.

b- Establecimiento de unos criterios de representación gráfica esenciales, tanto en las plantas y perfiles como en los alzados de tipo estratigráfico y tipológico-estructurales.

c- Cumplimentación ordenada de registros de control:

- U nidades estratigráficas.

- Bol sas de materiales.

- R egistro de cortes.

- Registro gráfico.

- Registro fotográfico.

d- Utilización de un sistema de fichas apropiado:

- Ficha de unidades única para cualquier tipo de el emento (emergente o soterrado).

- Ficha de control de obras.

- Ficha de control tipológico.

- Ficha de Muestreo edilicio.

- Ficha de estado previo.

Por lo que se refiere al EQUIPO BÁSICO Y RELACIÓN INTERPROFESION AL:

a- Selección de los miembros esencial es del equipo y sus actividades.

b- Los estudios multidisciplinares. Selección y val oración. Dentro del conjunto de actuaciones a realizar, el estudio de paramentos, en sus diferentes vertientes, supone la pieza fundamental, siendo además la línea de investigación en la que mayores effuerzos metódicos hemos invertido.

Dentro del sistema, los estudios de paramentos se ejecutan en dos niveles consecutivos de actuación. En primer lugar, como parte del período inicial de auscultación de la estructura se procede a las siguientes operaciones: - Obtención de un fichero completo en el que se reflejará el estado previo del edificio antes de la rehabilitación. Se realizará estancia por estancia, centrando nuestra atención en solerías, muros, vanos, detalles decorativos, etc.

- Identificación numérica de los ámbitos o estancias y numeración de los "Paramentos G uía", o al ineaciones principal es que conforman las crujías y compartimentos. Serán las primeras unidades estratigráficas de la lista que luego continuaremos. Podríamos optar por una jerarquización de las unidades tal y como propone Brogiolo (1988), pero pensamos que este tipo de disquisiciones nos alejan más que ayudarnos en el objetivo general de la comprensión global del inmueble. 
- Tras esta identificación, se procede al análisis de los distintos "tipos de adosamiento"; estos pueden ser de distinto tipo: simple, con encastres simples o complejos, coetáneos, etc.

Con ello, obtendremos una primera planta con una lectura general clara. Por lo general, el orden de los adosamientos y su tipología suele definir con muy pocos cambios los procesos constructivos del palimpsesto. La comprensión del modo en que contactan cada una de las alineaciones principales que configuran el esqueleto del inmueble, es indispensable para empezar a tener un dictamen sistemático de su evolución.

Para poder real izar esta lectura inicial debemos acceder a la fábrica de los muros en las zonas de unión. En el caso de que existan obras de arte, pinturas o impedimentos de cualquier tipo para poder descarnar y llaguear el muro, deberemos acudir a la excavación 0 a la interpretación de los contactos en la techumbre, y si esto tampoco fuera posible, recurrir en última instancia a la habitual reflexión analógica. Sin embargo, y siempre que sea posible deben iniciarse los trabajos con el llagueado de al menos un metro cuadrado continuado, o varios puntos separados; este picado debe real izarse, por supuesto posteriormente a un programa de catas en los enlucidos que descarte cualquier posibilidad de destrucción de pinturas 0 cualquier otro elemento paramental de interés artístico 0 arqueológico.

Posteriormente se identifican las divisiones edilicias general es; es decir, se procede a rellenar sobre una planta con las unidades paramentales guía, aquellos componentes fundamentales del muro: tapiales, ladrillo, piedra, mixtos, etc.

Con ambos estudios, el de adosamientos y ésta identificación de fábricas esencial es, ya pueden establecerse las primeras hipótesis de trabajo. Éstas, deberán plasmarse en una planta secuenciada en la que se otorgue a cada fase constructiva una trama distinta, acompañada de una matriz $\mathrm{H}$ arris interpretativa, con la secuencia cronológica provisional.

El siguiente nivel de aplicación se da en la fase de investigación propiamente dicha. En ella se procede a cumplimentar varios "ficheros de control arqueológico", destacando, a nivel paramental el Fichero tipológico y el de muestreo edilicio.

Respecto a ambos tipos de control, el uso de fichas específicas se hace imprescindible debido a varias razones. En el de M uestreos Edilicios, se ficha cada tipo distinto de aparejo, analizando a fondo sus elementos y relacionándolos estratigráficamente con otras unidades adyacentes, adjuntando número de muestra, medidas medias de los distintos el ementos configurantes, esquemas de ubicación de la muestra, etc. En el Fichero tipológico, con la intención de completar las tipologías edilicias, se fichan por tipos cada vano, arco, suelo, cimiento, aparejo, etc. enfatizando las cuestiones métricas y en general todos los paral el os y cronologías asociadas en otros edificios locales.

Seguidamente, tras la catalogación, se acometen los estudios paramentales sistemáticos (figs. 1, 2, 3, 4, 5). A nuestro entender existen dos vías de lectura de al zados, una con carácter estratigráfico y final idad evolutiva derivada de los estudios arqueológicos tradicionales (medievalistas ingleses, etc.) pero sobre todo desarrollada desde la aplicación del método $\mathrm{H}$ arris (es la representada por Parenti) (PARENTI, 1988); y otra con carácter analítico estructural, con finalidad descriptiva, tipológica y patológica (representada por Doglioni) (DogLIONI, 1988: 223-248). Existe una tercera vía analítica puramente arquitectónica (J IMÉNEZ, 1982), caracterizada, como ya vimos, por una minuciosa indagación física fundamentada en los aspectos tipológicos, descriptivos y espaciales.

En nuestro caso, la experiencia sevillana reciente ha ido derivando hacia la absorción de las distintas vías, vinculándolas al resto de operaciones arqueohistóricas bajo una misma óptica y final idad, asumiendo los fundamentos evolutivos, tipológicos, estructurales, etc., como diferentes caras de una misma moneda cuyo fin esencial es la val oración histórica de la estructura.

Por lo que respecta a los anál isis de tipo "EV O LUTIVO", nuestro sistema propone el seguimiento del sistema $\mathrm{H}$ arris simplificado, general izado en la medida de lo posible o circunscrito a aquellos muros que resulten básicos. Para ello, se procederá inicial mente a la citada estrategia de picado de paramentos, Il agueados o limpiezas de determinados el ementos. Cada al zado será di bujado de manera individualizada a escala 1:50 preferentemente. Para identificarlo se le dotará con su número de estancia y su orientación cardinal, además de la referencia a la planta. El registro de las unidades estratigráficas se cumplimentará mediante la ficha estratigráfica diseñada para el Cuartel del Carmen (TABALES, 1992), especialmente transformada para incoporar mejor los datos de alzados.

En cuanto al modo de representar, se realizarán dibujos exactos de los contornos de las unidades principales y simplificado de las secundarias. Esto es variable según el caso, tendiéndose a la esquematización a medida que subimos la escala. Se esquematizarán los interiores de las unidades (fábricas murarias, rell enos, tapiados) salvo cuando presenten características particularmente interesantes, 


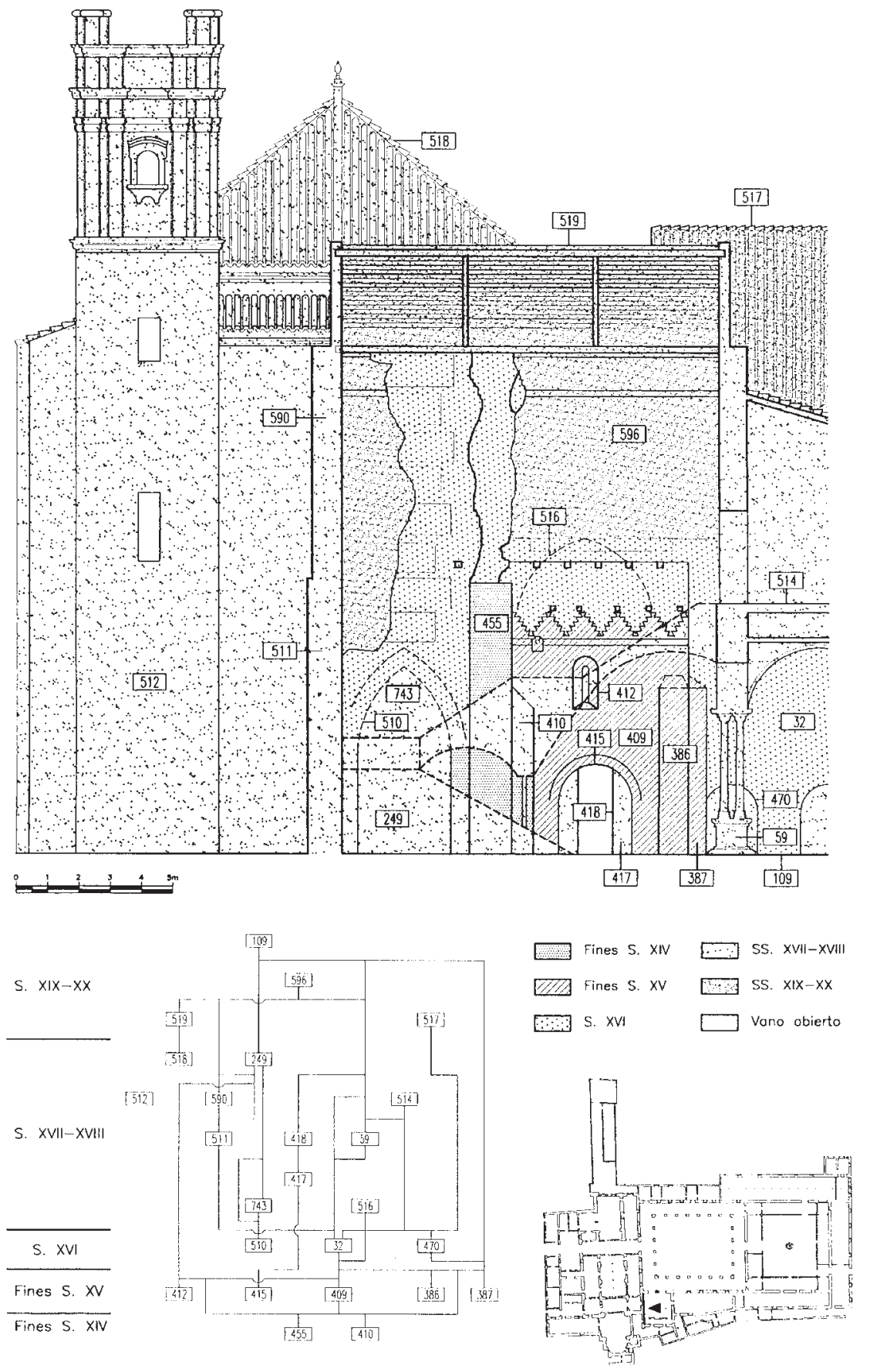

Fig.1. Análisis estratigráfico. Cuartel del Carmen (1990) 


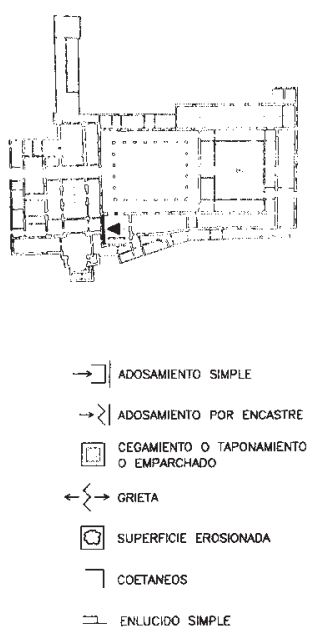

APAREJO VANOS

(13) SOGA Y TZON

(114) RREGUIAR

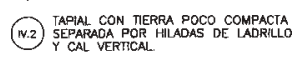

ENLUCIDOS

今 ENLUCIDO DE CAL SIMPLE

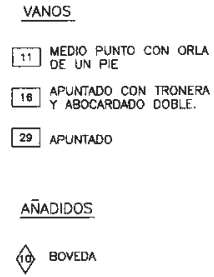

(1.) BOVEDA
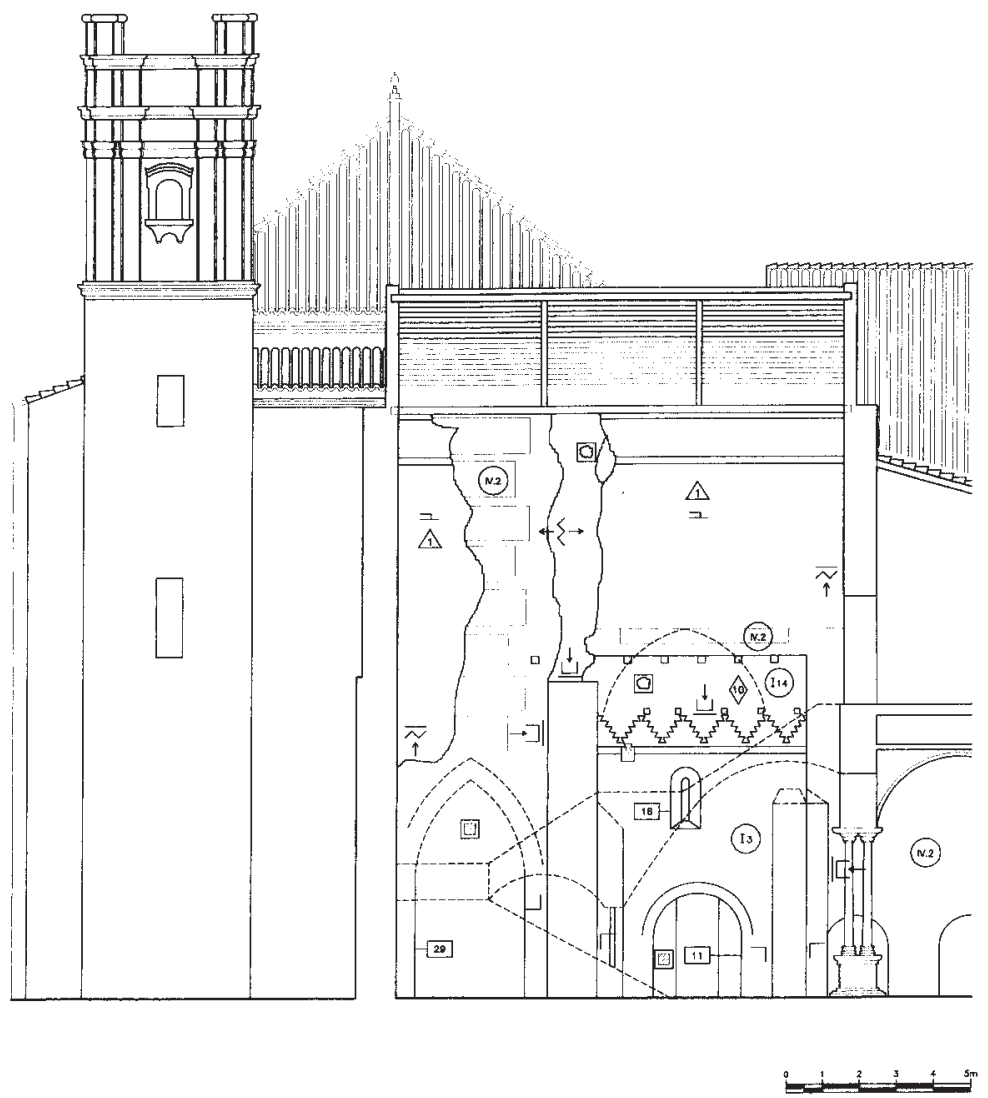

Fig.2. Análisis tipológico y descriptivo. Cuartel del Carmen (1992)

como en el caso de las reformas de las fábricas de muros. Cada fase, período o proceso constructivo será identificado mediante tramas. Preferimos simplificar los eventos cronológicos en un número reducido de fases mucho mas comprensibles.

Las unidades principales se numerarán simplificando en un número aquellas que forman parte de un grupo homogéneo y coetáneo, como los mechinal es de un forjado o las vigas de un techo. A este respecto la simplificación del método $\mathrm{H}$ arris, al igual que la excavación, nos permite concentrar nuestra labor de investigación en la globalidad del edificio.

LOS estudios "ESTRUCTURALES" fueron incorporados a nuestro sistema de análisis en el Cuartel del Carmen (1993) y desarrollados final mente en El Pal acio de los M arqueses de M archel ina (1995). Fruto de la cotejación de las experiencias propias (J IMÉNEZ, 1992) y foráneas (sobre todo DOGLIONI, 1988) relativas a la identificación de eventos no estratigráficos, pudimos comprobar cómo gran parte de las apreciaciones tipológicas referentes a los aparejos, o las patológicas (grietas, combamientos, rupturas estructural es 0 superficiales, etc.) incidían de manera providencial en la val oración arqueológica general (eincluso estratigráfica) (fig. 3).

Ante esta apreciación optamos por crear un modelo propio de indagación en ese campo, tradicional mente vetado a los arqueólogos, materializándose en varias tipol ogías fruto de la comprobación científica durante la úl tima década en excavaciones urbanas en la ciudad de Sevilla. $\mathrm{N}$ i que decir tiene que se trata de un model o embrionario que tenderá a ser transformado y mejorado a medida que se vayan incorporando nuevos datos.

Se dividen en dos:

1- Estructurales: Estableciendo mediante una simbología desarrollada por M.A.Tabales, (pero basada en DoglionI, 1988) las relaciones físicas más importantes (rupturas, erosiones, adosamientos, grietas, etc.).

2- Tipológi cos: Mediante un código creado al efecto, se identifican: aparejos, vanos, añadidos y enlucidos.

En el caso del aparejo, se subdivide en 4: el ladrillo (TIPO I); con una subdivisión en tipos basadas en Clairac y Parenti, añadiendo tipos sevillanos identificados hasta el momento. La Piedra (TIPO II); siguiendo a Parenti, complementados por M. A. Tabales. Los Mixtos (TIPO III); 
siguiendo a Parenti, complementado igualmente. El Tapial (TIPO IV); 14 subtipos (de momento) identificados en Sevilla por M. A. Tabales, en función de sus características formales. A este respecto, la experiencia aún es muy escasa como para val orar mej or los aspectos derivados de la composición de los cajones (aunque estamos en ello), mientras que los aspectos métricos atraviesan por una fase de indagación que posiblemente desemboque en nuevas tipologías en breve. En el caso de los vanos, hemos establecido una clasificación basada en nuestra experiencia en Sevilla al igual que en los enlucidos.

Los principios que justifican el sistema expuesto son los siguientes:

1. Vinculación esencial entre el estudio arqueológico y la rehabilitación; es decir, el análisis debe contemplarse integrado dentro de la renovación del inmueble. No debe entenderse como una indagación previa y desconectada del resultado final.

2. Comprensión diacrónica del edificio. Éste, como organismo que ha ido transformándose, se rige por unas pautas procesuales que deben ser captadas como prioridad básica de la investigación. En un segundo plano estarán las distintas lecturas en extensión de cada fase constructiva; no se debe dar prioridad a procesos concretos si antes no se ha desentrañado la secuencia completa ya que esto al ejará a la arqueol ogía de su principal justificación dentro del engranaje de la rehabilitación.

3. Inserción geohistórica y tipológica en el entorno. El edificio se encuentra imbuido dentro de una lógica urbanística e histórica. En las actividades del investigador debe estar presente el estado científico de la cuestión del entorno, única garantía de la val oración correcta de las evidencias rescatadas.

4. A nálisis general del inmueble. La parcial idad en la investigación es contraproducente en todos los nivel es y terminará por convertir en anecdótico lo que debe ser el armazón de las soluciones de rehabilitación posteriores.

5. El sistema de análisis arqueológico debe ser ágil y sistemático. Para aspirar a comprender un inmueble histórico desde una óptica secuencial debe tenerse en cuenta la vertiente estratégica de la investigación. D entro de ella se propone avanzar hipótesis que determinen la posterior indagación sistemática y para ello se deben real izar aproximaciones previas claramente prefijadas.

6. A tención prioritaria a la arquitectura. Al investigar edificios en pie el análisis sistemático debe partir de una serie de actividades preferentes entre las que destaca el estudio de lo elevado ya que éste será el objeto fundamental de la rehabilitación. Las operaciones constructivas se verterán sobre lo que permanece y por tanto no deben dejarse de lado los aspectos edilicios o estratigráficos esencial es para el posterior diseño. La excavación no es un complemento sino un pilar básico, pero por lo general su implicación real con la rehabilitación será menor.

7. A nálisis selectivo primando lo general sobre lo anecdótico. Las posibilidades de estudio histórico sobre cual quier edificio son muy variadas; dependerá del tipo de trabajo a realizar el adoptar una estrategia determinada dentro de la cual debe existir una premisa prioritaria: no ahondar en la recogida de datos hasta el punto de perder posibilidades efectivas de adquirir una interpretación global de la secuencia.

8. Mantenimiento de la investigación mientras existan remociones 0 alteración de lo preexistente, para lo que se adoptarán sistemas de análisis eficaces que propicien la recogida sintética del registro aunque la intervención arqueológica sistemática haya concluido. Si dicha actividad no se contempla el arqueólogo quedará excluido del proceso de toma de decisiones final.

9. Debe existir una coordinación del arqueólogo director de la investigación sobre los restantes estudios multidisciplinares: históricos documentales, analíticos, etc. que redunden en la comprensión histórica final. Sól o una multidisciplinaridad dirigida en la misma línea de lo argumentado hasta ahora (sel ectividad, gradualidad, etc.) justificará una vinculación real y no anecdótica con el proceso de obra.

10. Capacidad consultiva en la rehabilitación. No en el sentido de asumir competencias para la cual no existe formación en el campo de la arqueología, pero sí de garantizar la seriedad de los resultados mediante la colaboración entre el científico y el diseñador, de una manera reglada. Desde el col ectivo de arquitectos y sobre todo de arqueólogos debe entenderse que la irrupción de la arqueología en la rehabilitación es un fenómeno imparable que probablemente transforme a la larga las responsabilidades legales a la hora de proyectar.

\section{CONCLUSIÓN}

Esta revisión pretende mostrar una experiencia, la sevillana, tan válida como cual quier otra, confusa y contradictoria como pocas pero a la vez rica y entusiástica desde los inicios. Somos conscientes de que nuestra percepción de este asunto puede ser diferente de la de otros investigadores local es e incluso de la de otros grupos profesionales; la discutible autoridad que el que suscribe pueda tener a la hora de arrogarse una visión "histórica" de hechos y situaciones tan recientes, viene excl usivamente de su dedicación 


\section{PROPUESTA DE SIMBOLOGIA PARAMENTAL}

SIMBOLOS PARAMENTALES. (RELACION ENTRE UNIDADES Y CARACTERES)

$\rightarrow$ DIRECCION DEL ADOSAMIENTO.

$\rightarrow]$ adosamiento a bloque o con algun ENCASTRE.

$\rightarrow\langle|$ ADOSAMIENTO CON ENCASTRE.

COETANEIDAD.

$\zeta$ RUPTURA DE LA SUPERFICIE MURARIA.

$\square$ ENLUCIDO SIMPLE

$\sqsupseteq$ SUPERPOSICION DE ENLUCIDOS.

P] TAPONAMIENTO, CEGAMIENTO DE VANO O EMPARCHADO

(3) HUECO, MELLA O SUPERFICIE EROSIONADA

$\leftarrow \zeta \rightarrow$ GRIETA-FISURA.

888 UNIDAD ESTRATIGRAFICA.

$\stackrel{+0,15}{\nabla}$ COTA

- - D. DISCONTINUIDAD.

GROSOR MAXIMO: CONTORNOS ESTRUCTURALES

GROSOR MEDIO: CONTORNOS INTERNOS A DIFERENTES

PLANOS E INTERFACIES.

GROSOR MINIMO: DESPIECES ESTRUCTURALES O SOLERIAS.

SIMBOLOS TIPOLOGICOS
(13) TIPO DE APAREJO.
TIPO DE ENLUCIDO.
1. TIPO DE VANO.
(1) ElEMENTOS AÑADIDOS. 
profesional y metodológica al tema durante los últimos años. En ningún caso se pretende marcar caminos ni establecer sistemas infal ibles ya que todo lo dicho hasta ahora es revisable, discutible y si se quiere desechable $e^{3}$.

En "nuestra opinión" la conexión natural entre la investigación arqueológica específica y la rehabilitación del inmueble ha evolucionado mediante la interacción de varios conceptos básicos:

- La presencia de la arqueología en la rehabilitación, y su vinculación metodológica con los proyectos arquitectónicos. En este sentido se ha pasado de la nula incidencia a la integración formal. Ha sido esta una evolución con altibajos que no obstante, no se ve aun reflejada en una normativa general clara. De hecho, salvando la inevitable investigación en subsuelo, algo que sí recogen las cartas del riesgo de los distintos cascos históricos andal uces, la profundización en el conocimiento arqueológico de la edificación en sí ha tenido que ver más hasta ahora, con el propio interés del arquitecto o con la preparación del arqueólogo, que con el desarrollo de un modelo obligatorio. A pesar de ello hoy día, a diferencia de los ochenta, es impensable que se plantee una rehabilitación en el casco histórico con un proyecto en el cual la indagación arqueológica no esté garantizada. Las comisiones patrimoniales y la actuación vigilante de las distintas administraciones (Gerencia municipal de urbanismo y J unta de Andal ucía) son un aval para ello. Y a pesar de todo, en obras menores de rehabilitación en las que no existe movimiento de tierras pero sí trabajos emergentes que, como los picados murarios o consolidación de forjados, suponen una al teración de lo preexistente, la presencia arqueológica es mínima.

- El ámbi to de actuación. Se ha pasado de la excavación puntual a la lectura paramental integral y a la selección de modal idades de excavación en función de los objetivos, a la par que se organizan los control es de obra. Los trabaj os más antiguos, planteados como emergencias o urgencias arqueológicas, se caracterizaron por la apertura de un número de sondeos excesivo, y tal vez en parte innecesarios, y fueron causantes de no pocos problemas de estabilidad para el edificio en rehabilitación. Con el tiempo se han

\footnotetext{
${ }^{3}$ De hecho, en la actualidad, el grupo de Investigación sobre el Patrimonio Edificado del Departamento de Construcciones Arquitectónicas 2 de la Escuela Universitaria de Arquitectos Técnicos trabaja sobre nuevas clasificaciones tipológicas que enriquecerán en un futuro cercano nuestra visión de los inmuebles históricos en obra.

${ }^{4}$ En nuestra ciudad se han puesto en práctica recientemente otros sistemas similares a los empleados habitualmente en el resto de España. Edificios públicos y privados han contado con vigilancia e incluso estudios arqueológicos que han optado de nuevo por la preeminencia del subsuelo complementada con al gunas aproximaciones estratigráficas a lo emergente.
}

diseñado proyectos más lógicos y menos agresivos en los que la lectura de alzados ha supuesto un esfuerzo superior al de la excavación y ésta se ha centrado en actuaciones que combinaron el interés científico con las necesidades del edificio en sí y de la obra. $\mathrm{Ni}$ que decir tiene que para lograr una síntesis digna se dieron numerosos pasos en falso ya que ningún profesional vinculado a esta actividad (arquitectos, arqueólogos, historiadores del arte, restauradores) tuvo formación al guna previa que no fuera la del día a día en la obra. Los arqueólogos dejaron de mirar hacia abajo para incorporarse al andamio y tuvieron que asumir que el conocimiento puro del monumento no tenía justificación si no era en el ámbito de una operación de salvamento en la cual él no era más que una pieza. Los arquitectos tuvieron que asumir la necesidad de conocer en profundidad antes de tocar el inmueble y para ello tuvieron que del egar cuotas de poder relativo. Insistimos no obstante en la misma matización del punto anterior; es decir, este panorama de indagación ha afectado a numerosos inmuebles de notable calidad durante las dos últimas décadas pero en otros de carácter menor aun hoy se sigue prescindiendo del estudio pormenorizado de lo emergente desde nuestra óptica sal vo que el arquitecto responsable esté sensibilizado con el patrimonio.

- La especificidad del método arqueológico. Se ha pasado de aplicar sistemas dispares en función de la formación artística, prehistórica o medievalista, del arqueólogo, a generar una estrategia clara basada en la experiencia local y sobre todo en la manipulación libre de los trabajos italianos y españoles precedentes, sobre la base del método $\mathrm{H}$ arris y la revisión de Carandini. Somos muy conscientes de las carencias del sistema expuesto en las páginas anteriores aunque creemos haber conseguido cierto orden y nivel de conocimiento de los inmuebles investigados mediante su uso y a pesar de que seguimos considerando su transformación, no creemos equivocarnos en sus fundamentos. Cuestiones como la real ización de campañas indagatorias previas, el planteamiento del estudio de paramentos según la estratigrafía, la tipología y la descripción constructiva y patológica, la contemplación de tres modalidades de excavación según los objetivos tras el estudio de alzados, etc. configuran una manera de entender el acercamiento arqueológico al inmueble que se ha mostrado hasta ahora cuando menos útil4.

- La incidencia legal. Se ha pasado de la presencia anecdótica del arqueólogo a la reglamentación de cautelas de lecturas de paramentos en la carta del riesgo local y en las normas de protección de inmuebles. Es este un caso único que hasta ahora afecta sól o a los sectores del centro histó- 


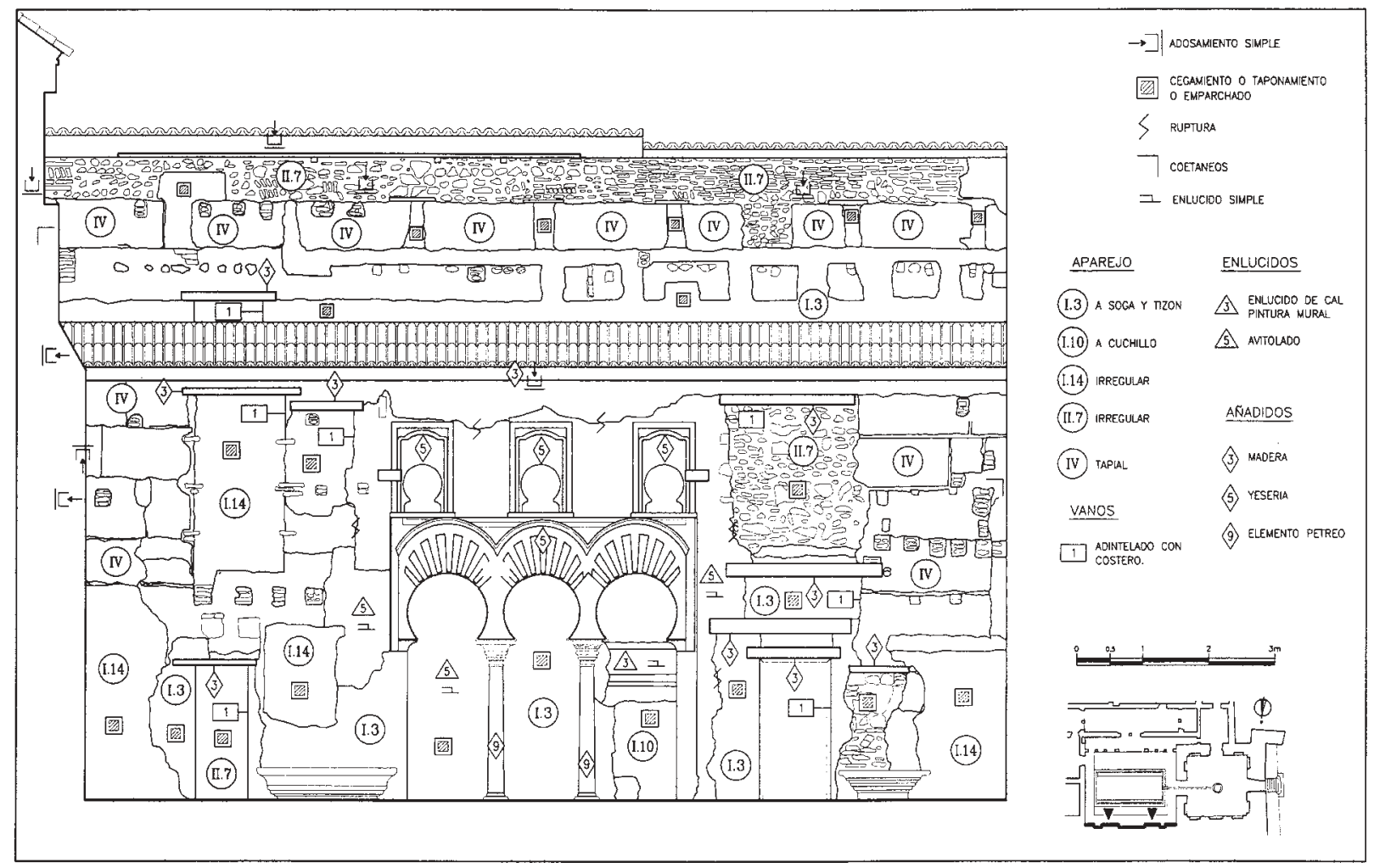

Fig.4. Análisis tipológico. Alcázar de Sevilla. Patio del Yeso (1998)

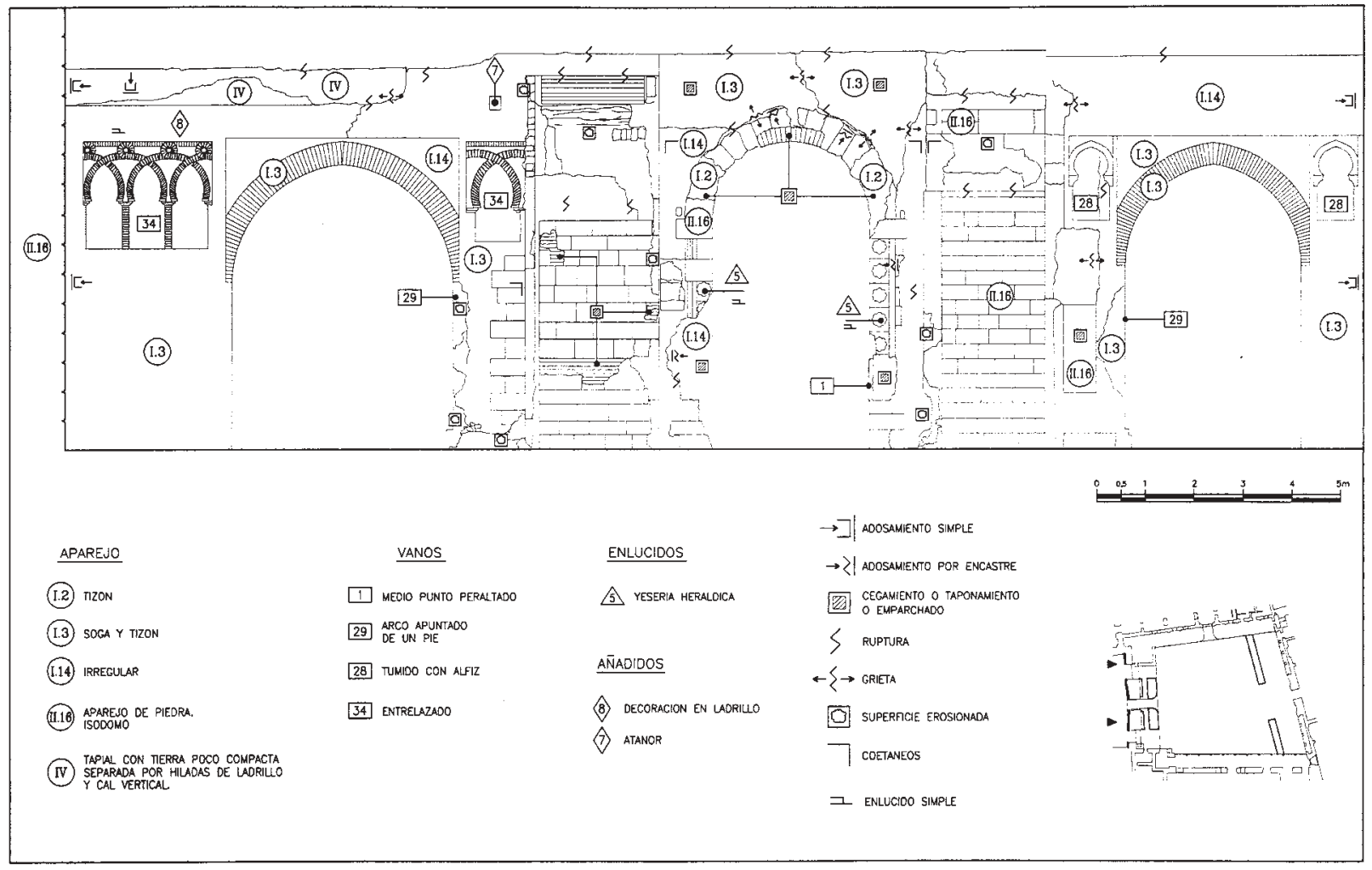

Fig.5. Análisis descriptivo. Alcázar de Sevilla. Patio del León (1997) 
rico en los que han entrado en vigencia los nuevos planes especiales de protección, todavía en proceso de ejecución. H asta el momento la gestión correspondiente a la presencia arqueológica en inmuebles estaba asignada a la Dirección General de Bienes Culturales de la Junta de Andalucía; de hecho así sigue siendo en gran parte del casco histórico donde a pesar de no existir norma al guna que obligue a dicha presencia cuando no hay remociones en subsuelo, sí existe de facto una vigilancia activa para evitar pérdidas irreparables. En un futuro cercano al gunas cartas del riesgo como las de Antequera, Málaga, Priego, Córdoba, etc. tienen previsto dotarse de cautelas si milares sobre la arquitectura emergente; estarán en relación con el sistema de Fichas Diagnóstico actual mente en el aboración por la Dirección G eneral de Bienes Cultural es ${ }^{6}$.

- La organización de la D ocencia y difusión. De la nula presencia de la investigación del edificio con criterios arqueol ógicos se ha pasado a la impartición de asi gnaturas como la de Análisis Arqueológico de Edificios, de la Escuel a U niversitaria de A rquitectura Técnica, así como su incorporación en Masters y cursos de doctorado7. En un futuro cercano está prevista la creación de una asignatura de Arqueología de la A rquitectura en la Escuela Superior de A rquitectura a cargo del catedrático A Ifonso Jiménez. $N$ o debe extrañar que sean las escuelas de A rquitectura las que hasta el momento se han interesado por la investigación arqueológica del inmueble ya que son las que surten a las rehabilitaciones de profesionales cada vez más interesados en el patrimonio, que aunque no serán los responsables de la investigación arqueológica, sí serán los principales receptores de sus informes. Sí sorprende no obstante que en las Facultades responsables de la formación de arqueólogos e investigadores capaces de desarrollar esta

\footnotetext{
${ }^{5}$ No se olvide que el Plan General de Ordenación U rbana de Sevilla, vigente desde 1988, cautela los edificios históricos y prohibe la destrucción de cual quier inmueble histórico lo cual supone, al menos en teoría, que no debe haber peligro de alteración grave. Otra cosa es que se altere la superficie muraria sin vigilancia o lectura arqueológica. La labor de la Delegación Provincial de Cultura hasta ahora ha sido modélica en dicha sal vaguardia.

${ }^{6}$ Los servicios de Conservación, Protección eInvestigación de la DGBC trabajan en la elaboración de una catalogación de inmuebles en la que esté presente con todo detalle el análisis arqueológico y documental previo a cualquier trabajo de recupe ración. D ebemos destacar la labor en este sentido del arquélogo del $D$ epartamento de Proyectos del Servicio de Conservación Arturo Pérez Plaza, así como de un nutrido grupo de arqueólogos y arquitectos de los tres citados servicios.

${ }^{7}$ El caso de la asignatura de Análisis de Formas y luego de Análisis Gráfico Arquitectónico impartidas en la ETSA desde 1973 es especial debido a su desvinculación respecto a la disciplina arqueológica actual y al desuso que de ella hacen los arquitectos locales, mayoritariamente no interesados en lecturas tan complejas como las realizadas por P. R odríguez o Alfonso J iménez.

${ }^{-}$Se puede producir la paradoja de que los arquitectos y aparejadores de una obra de rehabilitación tengan más conocimientos de la nueva disciplina que los arqueólogos encargados de la investigación.
}

disciplina, no exista conciencia de la falta de preparación en materias relacionadas con el conocimiento de la construcción antigua o los métodos específicos de indagación. Queremos creer que también en los departamentos de Arqueología se resolverá en un futuro este problemå.

- La aceptación social. Del rechazo manifiesto de la Arqueología, generalizado entre los organismos implicados en la rehabilitación se ha pasado en pocos años a la presencia de equipos organizados en las principales instituciones (Alcázar, Catedral...). Lo cierto es que en una ciudad donde los conflictos motivados por la arqueología de urgencia son cada vez más agudos parece existir al gún consenso en dicha actividad, precisamente por influir de modo directo en la recuperación y val oración de inmuebles claramente asumidos por el colectivo como símbolos de su pasado.

En síntesis, si de al go sirve nuestra experiencia local es tal vez de ejemplo de integración gradual entre las necesidades científicas y los procesos de obra, debido sobre todo a una constante fricción metodológica cuyo resultado, al menos momentáneamente, es esperanzador. $\mathrm{N}$ os consta que en otras comunidades, provincias y ciudades se comienzan ahora a dar pasos similares a los que se dieron aquí años atrás, planteándose los mismos problemas que ya eran discutidos en los congresos ital ianos de los años setenta y ochenta, y repitiéndose los mismos planteamientos poco difundidos debido al carácter local y atomizado de nuestras publicaciones.

Sirva este foro de Vitoria como verdadero aci cate para difundir y generalizar de una vez por todas las múltiples experiencias desperdigadas entre congresos, memorias y artículos sin apenas difusión.

\section{Bibliografía}

Brogiolo G. P., 1988, A rcheologia del' 'edilizia storica, Como.

CABALLERO L., 1985, La intervención arquitectónica anal izada desde la intervención arqueológica, en A ctas del VII cursillo sobre la intervención en el patrimonio arquitectónico. Vic 1984. Barcel ona.

CABALLERO L., 1987, El método arqueológico para la comprensión del edificio. Dualidad sustrato-estructura, Curso de mecánica y tecnol ogía de los edificios antiguos. COA M. Madrid, pp. 13-58.

CABALLERo L., 1995, M étodo para el análisis estratigráfico de construcciones históricas o "Lectura de paramentos", Informes de la construcción 46, no 435, Madrid, pp. 37-47.

CARANDINI A., 1977, Per una carta dello scavo archeologico, A rcheologia M edievale, VI, Firenze.

D OGLION I F., 1988, La ricerca sulle strutture edilizie tra archeologia stratigrafica e restauro architettonico, en R. FRAN COVICH, R. PARENTI (a cura di), A rcheologia e Restauro de monumenti. I ciclo di lezioni sulla ricerca applicata in archeologia. Certosa di Pontignano (Siena) 28 settembre 10 ottobre 1987, Firenze, pp. 223-246.

FRAN COVICH R., 1979, Alcuni problemi dei rapporti pratici fra Archeologia, Restauro e Pianificazione Territoriale, A rcheol ogia M edievale, VI, Firenze. 
Gon ZÁlEZ A., 1985, Recerca i disseny. El monument com a document historici com objecte arquitectonic viu, Barcel ona

JIMÉNEZ A., 1992, La puerta de Sevilla en Carmona, Sevilla.

LÓPEZ A., 1984, Consideraciones metodológicas sobrela actuación del Servicio de Catal ogación y Conservación de M onumentos en el campo de la investigación arqueológica, en A ctas de VII cursillo sobre la intervención en el patrimonuio arquitectónico, Vic, Barcelona.

OJEDA R., TABALES M. A., 1996, La investigación arqueológica en Bienes Inmuebles. Metodología aplicada en la Puerta de Córdoba de Carmona, B oletín del Instituto A ndaluz de Patrimonio H istórico, 15, Sevilla.

OLIVA D., 1993, La investigación en lo construido como apoyo a la restauración del Patrimonio Arquitectónico, en R etauración Casa-palacio de M iguel de $M$ añara, Sevilla.

PARENTI R., 1988, La tecniche di documentazione per una lettura stratigrafica dell'elevato, en R. Francovich, R. Parenti (a cura di), A rcheologia e Restauro de monumenti. I ciclo di lezioni sulla ricerca applicata in archeologia. Certosa di Pontignano (Siena) 28 settembre10 ottobre 1987, Firenze, pp. 249279.

TABALES M. A., 1993, M etodología arqueológica aplicada a la rehabilitación de edificios históricos. Intervención en el Convento de Santa M aría delos R eyes de Sevilla. Tesis de Licenciatura inédita. Depto. De Prehistoria y Arqueología. Universidad de Sevilla.

TABAlEs M. A., 1997, La Arqueología en edificios históricos. Propuesta de intervención y análisis global a través de la experiencia sevillana, B oletín de Instituto A ndaluz del Patrimonio $\mathrm{H}$ istórico, 20, Sevilla.

TABALES M. A., 1998, A rqueología y Rehabilitación de edificios históricos en Sevilla. Tesis Doctoral. Departamento de Prehistoria y Arqueología. Universidad de Sevilla.

TABALES M . A ., 1999a, A nál isis arqueológico de paramentos. A plicaciones en el patrimonio edificado sevillano, Spal, rev. de D epto. de Prehistoria y A rqueol ogía U niversidad de Sevilla.

TABALES M. A., 1999b, Análisis de paramentos en Sevilla, en V Congreso de A rqueol ogía M edieval Española, Valladolid.

TABALES M. A., 1999C, La arqueología y las técnicas constructivas medievales, en L a Construcción medieval, Escuela U niversitaria de A rquitectos Técnicos de Sevilla.

TABALES M. A., 2002, Sistema de análisis arqueológico de edificios históricos, Monografías del Instituto de Ciencias de la Construcción de Sevilla, Sevilla. 